\section{Unterstützung für ein Frauengesundheitshaus in Prishtina (Kosova)}

\author{
A. Mühlemann, E. Blaser
}

Die aktuelle Situation in Prishtina (Kosova) braucht Projekte, die den Wiederaufbau fördern und der Bevölkerung "Hilfe zur Selbsthilfe» bieten. Die Frauen in Prishtina zählen zu einer besonders gefährdeten Bevölkerungsgruppe. Viele Frauen sind alleinerziehend, erwerbslos und ihre gesundheitliche Versorgung ist schlecht.

Frau Dehari, eine albanische Krankenschwester aus Ex-Jugoslawien, welche seit mehreren Jahren in der Schweiz Übersetzungs- und kulturelle Vermittlungsarbeit geleistet hat, plant nun, als Projektleiterin ein Frauengesundheitshaus in Prishtina aufzubauen. Der Christliche Friedensdienst (cfd), ein schweizerisches Hilfswerk, prüft zurzeit die Möglichkeit, das Projekt längerfristig zu unterstützen und zu begleiten.

Viele Angehörige medizinischer Berufe, die in Bern mit der albanischsprechenden Bevölkerung zu tun haben, lernten Frau Dehari als umsichtige und kompetente Übersetzerin bei Behandlungs- und Beratungsgesprächen schätzen. Wir haben Frau Dehari 1997 im Rahmen unserer Tätigkeit als Assistenzärztinnen am Kriseninterventionszentrum der Uni-

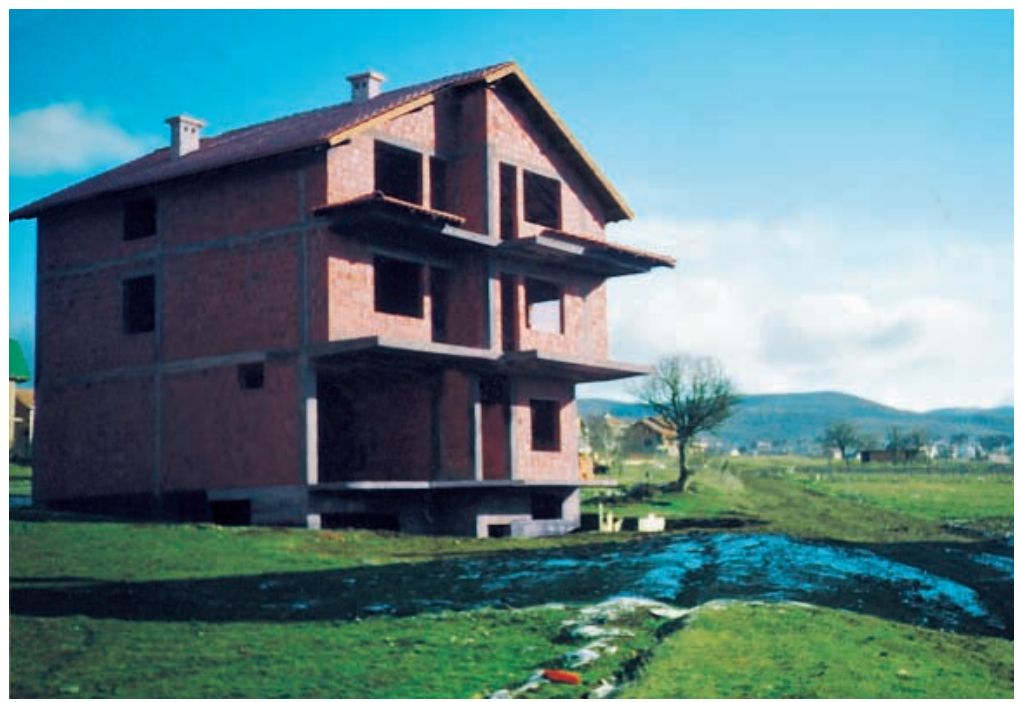

Korrespondenz:

Dr. med. A. Mühlemann

Giessereiweg 9

CH-3007 Bern versitären Psychiatrischen Dienste Bern kennengelernt. Die Zusammenarbeit hat sich später in unseren psychiatrischen Praxen weiterentwickelt.

Frau Dehari wird im Sommer 2001 mit ihrer Familie nach Prishtina ziehen. Das Projekt Frauengesundheitshaus wird dort von einer Arbeitsgruppe unterstützt, die aus einer Gynäkologin, einer Krankenschwester, einer Pharmaassistentin und einem Röntgenarzt besteht. Geplant ist die Vernetzung mit einer lokalen Organisation, welche das Projekt innerhalb von zwei bis drei Jahren selbständig übernehmen kann.

\section{Wäscherei, Ambulatorium und Apotheke in einem Haus}

Das Frauengesundheitshaus, dessen Rohbau bereits errichtet ist, soll den Frauen in verschiedenen Bereichen Unterstützung anbieten.

Im Keller des Hauses befindet sich eine kleine Wäscherei. Diese bietet Arbeitsplätze für vier bis fünf Frauen im Bereich Lingerie. Im Parterre und im ersten Stock befinden sich ein Frauenambulatorium und eine Apotheke. Das Frauenambulatorium ermöglicht gynäkologische Untersuchungen, Familienplanungsund Schwangerschafts- sowie generelle Gesundheitsberatung. Im Frauenambulatorium arbeiten zwei Ärztinnen, eine medizinische Praxisassistentin und eine kaufmännisch ausgebildete Person. Eine im Gesundheitshaus integrierte Apotheke ermöglicht den $\mathrm{Zu}-$ gang zu Medikamenten. Die Apotheke soll auch Produkte der Komplementärmedizin führen. In $\mathrm{Zu}-$ sammenarbeit mit den Ärztinnen sollen Kurse und Schulungen zur Gesundheitsvorsorge und zum Gebrauch von Heilmethoden wie Massage, Wickel usw. durchgeführt werden.

\section{Spenden gesucht}

Um das Projekt verwirklichen zu können, braucht es dringend finanzielle Unterstützung durch Geldspenden.

Für den Start des Projektes werden jedoch auch Einrichtungsgegenstände und medizinische Geräte benötigt und gesucht.

1. Für das gynäkologische Untersuchungszimmer: sämtliche Einrichtungen von der Untersuchungsliege bis zum Spekulum.

2. Für allgemeinärztliche Untersuchungen: sämtliche Einrichtungen (EKG, Blutdruckmesser, Thermometer usw.).

3. Sterilisationsapparat.

4. Ultraschall- und Röntgengerät.

5. Aggregator für Stromausfälle.

6. Für die Apotheke: Mobiliar und Einrichtungsgegenstände.

Geldspenden überweisen Sie bitte auf das Bankkonto: Valiant Bank (21), 3001 Bern, Konto 30-38112-0, Dzemile Dehari-Sulejmani. 
Falls Sie medizinische Geräte oder Einrichtungsgegenstände spenden können oder Fragen bezüglich des Projektes haben, setzen Sie sich doch bis zum 17. Juli 2001 direkt in Verbindung mit: Frau Dehari, bei Familie Krasnizi, Looslistrasse 68/8, 3027 Bern, Natel 0765140460.

\section{Michaele oder Der Himmel ist ein grosses Loch}

E. Danieli
Ab dem 17. Juli 2001 nimmt Frau Blaser Angebote gerne entgegen (Frau E. Blaser, Ärztin für Psychiatrie und Psychotherapie, Bollwerk 41, 3011 Bern, Tel. 03131827 47, Fax 03131827 48).

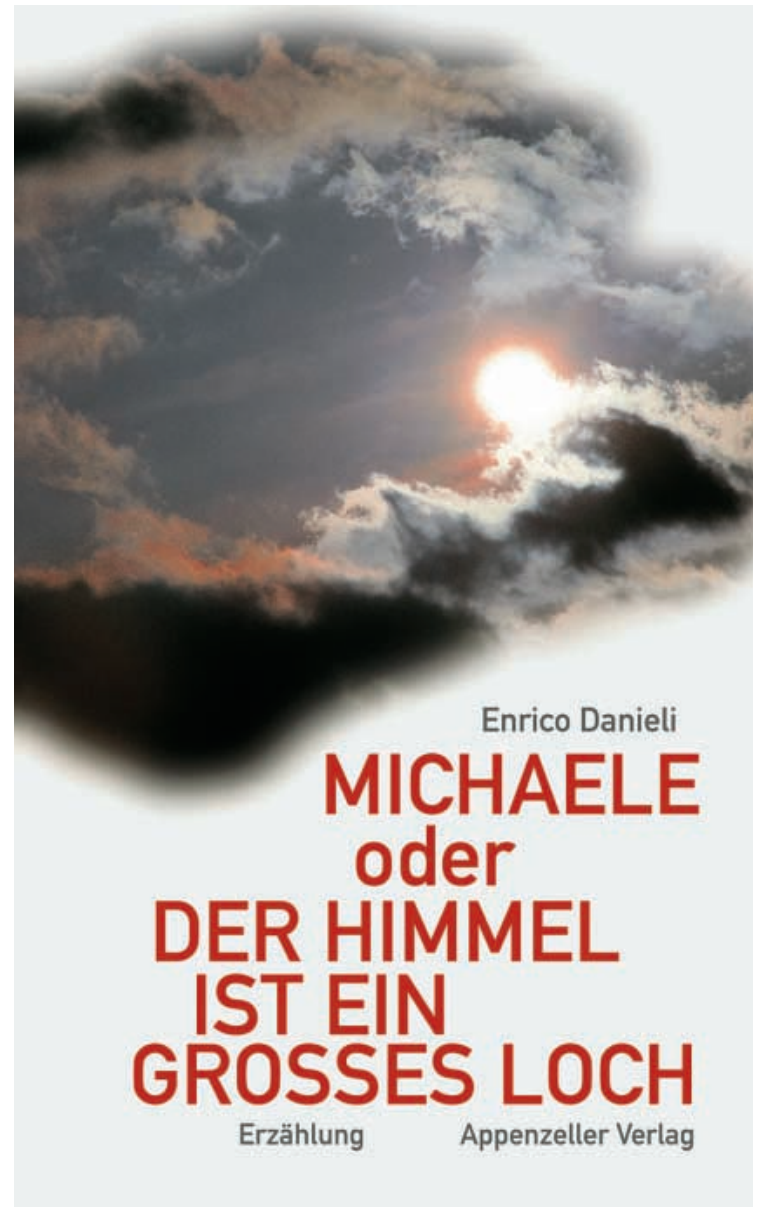

Kurz nach Beginn der Veranstaltung, nach der Begrüssung und Vorstellung des Gastreferenten Professor Tissot-Guerraz aus Lyon durch den Vorsteher des Departements für Neurologie, Professor Hess, störte ein Kranker, ein Epileptiker, hiess es, mit lautem Grunzen aus einer der hintersten Reihen die Vorlesung. Und als der Vorsteher der Klinik rief: "So holt doch einen Arzt!», löste das trotz des allgemeinen Unbehagens Heiterkeit aus. Der Störenfried wurde von mehreren Zuhörern an Armen und Beinen aus der Sitzreihe gezerrt und aus dem Saal entfernt. Die Anwesenden gaben mit einem Klopfen auf die Vorlesungspulte ihrer Zustimmung Ausdruck.

Michaele kam in Begleitung einer jüngeren Krankenschwester, die den unsicher und schwankend Gehenden stützte. Er trug einen dunklen Rollkragenpullover, weite Jeans und Turnschuhe und hatte sich 
neben dem Katheder auf einen bereitstehenden Stuhl zu setzen. Er sass da, als ginge ihn alles nichts an, während der berühmte Professor aus Lyon seinen Vortrag hielt: "La spongiose est l'élément le plus caractéristique. Il donne aux tissus nerveux un aspect d'éponge du fait de la présence de nombreuses petites cavités dites vacuoles ...", und während der Mann mit dem grossen, runden Kopf und einer an einer blauen Schnur festgemachten Hornbrille Lichtbilder projizieren liess, um den Zuhörern die Hirne der Kranken und der von ihm mitentdeckten neuen Krankheitsvariante zu zeigen, «... donnant un aspect d'éponge ... avec un anticorps anti-prion ... plaque amyloïde entourée de vacuoles de spongiose ... cet aspect floride est évocateur de la nouvelle variante ..." - nur kurz hatte Professor Hess unterbrochen und die im Deutschen gebräuchlichen Wörter eingefügt: "Rosetten, Totenladen" -, sass Michaele als einer aus einer anderen Welt unter uns. Auf seiner Stirn hatten sich Schweisstropfen gebildet und für einen Moment erinnerte ich mich, der ich weit hinten sass und die gezeigten, violett gefärbten Hirnschnitte mit den grossen, blasenartigen Aufhellungen nicht verstand, an den von Natalia geschilderten Auftritt in Allenwilen. Michaele, der, den Kopf gesenkt, seine Beine und Füsse nicht ruhig halten konnte, schien die spezielle Situation, in die er wegen seiner Krankheit geraten war, nicht wahrzunehmen.

Während der Professor weiter von EEG-Ableitungen ("Ils peuvent montrer des décharges périodiques d'élément bi- ou triphasiques permanents pendant des jours") sprach und im gleichen Rahmen vom "type pace-maker» berichtete, später Zuhörer zu diesen und anderen Themen Fragen stellten (von sogenannten Pleds war die Rede, von periodischen, lateralisierten epileptiformen Entladungen), rutschte Michaele auf seinem Stuhl immer tiefer, bis die Krankenschwester, aufmerksam genug, ihn mit einem gekonnten Griff wieder aufrecht hinsetzte. Kurz vor Ende des Gastvortrages, "l'incubation est souvent très longue, traiteusement silencieuse, pouvant quarante ans ... elle se manifeste surtout par un tableau de démence ... elle est fatale», wegen der Wärme im Hörsaal waren die Vorhänge hochgezogen und die Fenster geöffnet worden und das Luftzufächeln hatte ein Ende gefunden, war Michaele, schläfrig und abwesend, seitlich eingeknickt und nur mit Hilfe eines in der ersten Reihe sitzenden, älteren Mannes gelang es, den steifen Körper wieder aufzurichten.

"Ce cas ici», sagte nun Professor Tissot-Guerraz, und zeigte mit seinem Laserleuchtstift, dessen roter Punkt Michaeles Kopf zu durchbohren schien, auf den Kranken. "Dans le début des années 80, la fabrication d'hormone de croissance extractive d'origine humaine, du cadavre humain ..." Michaele, noch immer den roten Punkt in seinen Haaren, als hätte er gespürt, dass von ihm gesprochen worden war, setzte sich kerzengerade hin. Und seine typischen Gesten, eine Erinnerung an früher, wurden von ihm, wenn auch sinnloserweise, wieder ausgeführt: Haare aus der Stirne werfen, Augen reiben. Professor Hess hatte sich erhoben und dem Gast, der die Redezeit von dreissig Minuten offenbar schon überschritten hatte, zum Zeichen des Endes die Hand zum Dank gereicht, während das Auditorium freundlich und lang anhaltend klatschte.

Nun war Michaele an der Reihe, und ein junger Assistent in weissem, zugeknöpftem Mantel näherte sich ihm, während die beiden Professoren auf der Bank neben dem Katheder Platz nahmen. Die Krankenschwester, vorbereitet auf den Auftritt, half Michaele, den Stuhl zu verschieben. Er sass jetzt in der Mitte des Hufeisens und konnte den schwer gewordenen Kopf am Katheder abstützen. Sitzend sprach der französische Professor von "la nouvelle variante ... les patients sont jeunes ... les premiers symptômes ne sont pas cognitifs ... des signes psychiatriques ... des modifications de personnalité ... de dépression ... des épisodes délirants ou des hallucinations visuelles ... anomalie de la perception sensitive ... une insomnie est fréquente ... de troubles de l'équilibre ...» Er sprach mit monotoner Stimme weiter, und ohne von Michaele Kenntnis zu nehmen, schrieben viele der Zuhörer ununterbrochen mit. Michaele musste aufstehen und mit Hilfe ging er unsicher einige Schritte hin und her, während sein Kopf abgeknickt und unnatürlich nach hinten hing. Wiederholt erfasste ein Zittern seinen mageren Körper und ein Zucken ging durch seine Beine, und ich beobachtete, dass sich sein Pullover unter den Achselhöhlen schwarz verfärbte. Und, als bedürfte der berühmte Professor der Unterstützung, ergänzte oder übersetzte der Klinikleiter die wichtigsten Begriffe, und der Assistent machte sich mit Reflexhammer, Stimmgabel und kleinen, sich drehenden Rädchen und Nadeln an den Armen und Beinen von Michaele zu schaffen. "Ataxie ... Dysarthrie ... les yeux sont aveugles ... Muskelstarre und Faszikulationen ... le language est sourd et malentendu ... une voix hachée ..."

Der Kranke liess einer Puppe gleich alles mit sich geschehen und zitternd bewegten sich die Glieder auf Stimulation in auffälliger Art hin und her, und es zuckten seine Beine und Füsse und machten schmerzhafte Verrenkungen, aber alles ohne sein Mittun und ohne Gefühlsbeteiligung. Auf die Frage des Assistenten, ob er ihm nicht die Hände zeigen könne, wiederholte Michaele die Frage mit leiser Stimme. Fragte der junge Arzt, ob er ihm nicht die Füsse zeigen könne, antwortete Michaele in gleicher Weise.

Wegen der fortgeschrittenen Zeit musste die Untersuchung beendet werden, und Professor Hess gelang es, mit einem Bonmot - "Prionen», sagte er lachend, "haben nichts mit prier zu tun" - doch noch eine versöhnliche Note in die Veranstaltung zu bringen.

Mir drehten sich die Wörter coucher du soleil, vacuoles, voix hachée, on est seul aussi chez les hommes, baobab und Asteroide 325 und B 612 im Kopf, und ich war froh, dass ein Gongschlag die Vorlesung beendete. Als die Krankenschwester Michaele aus dem Raum geführt hatte, wurden der Professor und der Assistent mit einem lang anhaltenden Klopfen verabschiedet. Wie betäubt von der Wärme und vom Gehörten und Gesehenen, erhob sich das Publikum langsam und verliess den Saal in eigentümlicher Stille, nur der knarrende Holzboden war zu hören. 